\title{
A SIMPLE MOUNTING FOR DEMONSTRATION SLIDES
}

\author{
RICHARD E. SCAMMON \\ Institute of Anatomy, University of Minnesota \\ ONE FIGURE
}

The mounting and preservation of special and demonstration slides is sometimes a matter of considerable importance, particularly when the specimens are not easily duplicated. For the past year the mounting device shown in the figure below has been in use in this laboratory, and it has proved so useful that its description has seemed worth record.

The mounting consists of a card 3 by 5 inches-the size used in most cataloguing work. It is made of heavy white cardboard, approximately $2 \mathrm{~mm}$. thick. In the center of this card is cut a rectangular opening, 26 by $76 \mathrm{~mm}$., which will just accommodate an ordinary slide. The slide to be mounted is placed in the opening and strips of passe-partout paper, three-eighths of an inch in width, are pasted along its upper and lower edges and to the adjacent edges of the card. These strips are cut long enough so that they extend a little beyond the ends of the slide to the right and left. They are pasted on both the front and back surfaces of the card. Two more strips of passe-partout paper, cut to the proper length, are then pasted completely around the card from front to back in such a position that they will cover the ends of the slide and also the cut ends of the horizontal strips of paper which hold the upper and lower edges of the slide in place. In this way the slide is bound in position within the opening and is held securely along all of its edges.

Slides mounted in this way may be filed in ordinary cardcatalogue boxes and occupy but little space. The unoccupied portion of the card affords considerable space for a record of the specimen. 
Mounts of this kind are practically unbreakable in ordinary class use and, so far as our experience goes, they do not warp or crack. The cost of a single mount, including the price of the original die for cutting the slit in the card, is about two cents.

A device somewhat similar to the present one was recently described by Robinson. ${ }^{1}$ He uses a somewhat larger eard with

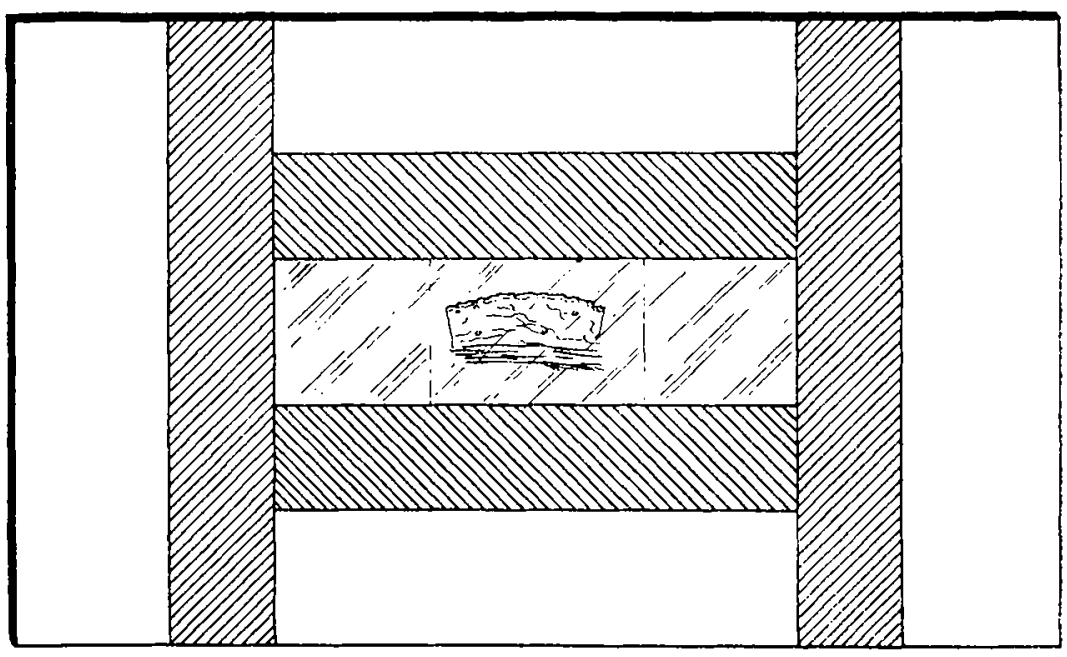

Figure 1

a circular opening cut through the lower part. The slide is fastened to the back of the card behind the opening. This mounting has the advantage of allowing more space on the card for a record, and is therefore particularly useful for autopsy material, but the mounting is not as secure as the one here described nor do the cards file so compactly.

${ }^{1}$ A convenient method for filing surgical sections. Jour. Am. Med. Ass., vol. $71, \mathrm{pp} .1379,1380$. 